\title{
ENFOQUES Y DIMENSIONES PARA EL DESARROLLO DE INDICADORES DE JUVENTUD ORIENTADOS A SU INCLUSIÓN SOCIAL Y CALIDAD DE VIDA
}

\author{
DINA KRAUSKOPF
}

\begin{abstract}
RESUMEN
En el presente artículo, se analizan los procesos específicos que deben contribuir al avance del posicionamiento de las juventudes dentro de las sociedades. Se identifican los enfoques y objetivos con que se abordan las políticas y los indicadores que apuntan a su inclusión y progreso de la calidad de vida. Se destaca que la fase juvenil es marginada de los indicadores de progreso de las sociedades y una de las más desatendidas del ciclo vital. Se revisan las perspectivas predominantes que obstaculizan la incorporación social de los jóvenes así como el problema de los criterios de edad en las políticas. A partir de enfoques incluyentes que favorecen la incorporación y calidad de vida de las juventudes, se proponen lineamientos en la construcción de indicadores.

PALABRAS CLAVE: JÓVENES, INCLUSIÓN SOCIAL, CALIDAD DE VIDA, INDICADORES, POLÍTICAS

* $\quad$ Profesora Emérita de la Universidad de Costa Rica. Académica de la Universidad de Chile. Miembro del Consejo Académico de la Maestría en Adolescencia de la Universidad del Desarrollo. Consultora Internacional en Políticas de Juventud.

Correo electrónico: dina.krauskopf@gmail.com.

Este artículo se basa en la presentación efectuada en el Encuentro de Investigadores Latinoamericanos: «La medición del progreso de las sociedades y el bienestar de sus habitantes, organizado por la OCDE y el Foro Consultivo Científico y Tecnológico de México».
\end{abstract}




\title{
ENFOQUES E DIMENSÕES PARA O DESENVOLVIMENTO DE INDICADORES DE JUVENTUDE ORIENTADOS PARA SUA INCLUSÃO SOCIAL E QUALIDADE DE VIDA
}

\section{RESUMO}

São analisados os processos específicos que devem contribuir para o avanço do posicionamento das juventudes dentro das sociedades. São identificados os enfoques e objetivos com que são abordadas as políticas e os indicadores que apontam para sua inclusão e progresso da qualidade de vida. Destaca-se que a fase juvenil é marginalizada nos indicadores de progresso das sociedades, sendo uma das fases mais desatendidas do ciclo vital. São revistas as perspectivas predominantes que impõem obstáculos à incorporação social dos jovens, assim como o problema dos critérios de idade nas políticas. A partir de enfoques inclusivos que favorecem a incorporação e a qualidade de vida das juventudes, são propostas diretrizes para a construção de indicadores.

PALAVRAS CHAVE: JOVENS, INCLUSÃO SOCIAL, QUALIDADE DE VIDA, INDICADORES, POLÍTICAS

\section{FOCUS AND DIMENSIONS FOR THE INDICATIONS OF DEVELOPMENT OF YOUTH ORIENTED TO SOCIAL INCLUSION AND QUALITY OF LIFE}

\begin{abstract}
This article analyzes specific processes that contribute to the advancing of position of youth inside of societies. It also identifies the focus and objective which aboard the political indicators that mark inclusion and progress in the quality of life. As well as highlighting that the juvenile phase is a marginal indicator of progress in the societies and one of the most unrecognized of the vital cycle. It revises the predominant perspectives that are obstacles in the incorporation of social youth as a problem of criteria of age in politics. The beginning including focus favors the incorporation and quality of life of youth which suggests alignments in the indicators of construction.
\end{abstract}

KEY WORDS: YOUTH, SOCIAL INCLUSION, QUALITY OF LIFE, INDICATORS, POLITICS 


\section{INTRODUCCIÓN}

LA INCLUSIÓN SOCIAL DE las juventudes exige la activa participación de todos los sectores y actores de la sociedad, al igual que la aplicación inmediata de políticas creativas que propicien su desarrollo. La complejidad de este cometido tiene diversas avenidas, entre las que está la clarificación de los enfoques y objetivos con que se abordan las políticas, y los indicadores que apuntan a su progreso son fundamentales, por lo que se tratan a continuación.

En el progreso de las sociedades están implícitos al menos tres niveles interactuantes: avances en el logro de la plenitud biológica, psicológica, social y económica (calidad de vida), avances en la equidad de la distribución de estos logros en la población y entre los países (profundización de las democracias), y estrategias para fomentar la consecución de estos dos objetivos (políticas públicas y programas). El progreso de la calidad de vida de las juventudes demanda, además de los procesos generales que permiten alcanzar el bienestar económico, tecnológico y material, otros procesos específicos que deben contribuir al avance en su posicionamiento dentro de las sociedades.

Para la mejora de la calidad de vida se requiere la inclusión de todos los actores sociales, el fomento de su capacidad de incidir en las transformaciones con una voz política y el combate a las diversas fuentes de desigualdad, por lo cual es necesario combatir la marginación de las juventudes. Contribuye a dicha marginación la existencia de un vacío en la profundización del análisis de los determinantes del bienestar de la población a partir del ciclo de vida y su impacto en las diversas generaciones que constituyen las sociedades del siglo XXI.

\section{LA IMPORTANCIA DEL CICLO VITAL EN LOS INDICADORES DE PROGRESO DE LAS SOCIEDADES}

Queremos señalar que los tres niveles para el logro del progreso que identificamos al comienzo de este texto, están atravesados por aspectos evolutivos del desarrollo de las personas en sus sociedades. El desarrollo integral a lo largo de su ciclo de vida se da en diversas dimensiones: biológicas, psicológicas, afectivas, espirituales y socioculturales. En el desarrollo de grupos o personas se presentan potencialidades, vulnerabilidades y múltiples posibilidades para modificar la realidad y ser modificado por ella. Las características se manifiestan de acuerdo a situaciones 
que aportan diversidad como la edad, el género, la condición económico-social, cultural, étnica, geográfica, etcétera.

UNICEF (2001) ha operacionalizado el enfoque de desarrollo en el marco de la división por edades del ciclo de vida desde la niñez hasta los dieciocho años. Indudablemente, estas categorías tienen sólo una finalidad orientadora, puesto que, como afirma Hart (1999) no pueden tenerse nociones universales de lo que son capaces las personas en las diversas fases de su desarrollo. Por otro lado, es un hecho que la edad - si bien es un dato insuficiente para dar cuenta de las fases que atraviesan los procesos del desarrollo- es un dato necesario en investigaciones, políticas, programas y legislación.

La utilización del enfoque de ciclo de vida es útil mas allá de la edad límite que UNICEF utiliza de acuerdo a su población meta siguiendo el mandato de la Convención sobre los Derechos del Niño (CDN), que establece que la niñez dura hasta los dieciocho años. Es necesaria la introducción de indicadores de juventud como etapa del ciclo vital, pues las personas jóvenes son cada vez más un período crucial en un rango relevante de años, dado el contexto de los cambios de la globalización y la modernización, particularmente la prolongación de la vida. La vejez es un período tan marginado como la adolescencia y la juventud. La adultez, en ausencia del enfoque de ciclo de vida, pasa a ser la edad predominante de la población en las sociedades en los indicadores de la medición del progreso.

Cuando se ha reconocido la falta de indicadores de progreso en juventud, se considera metodológicamente que es el mismo vacío que se presenta con comunidades culturales específicas como las etnias o sectores con situación diferenciada en la realidad social, como son los migrantes. Sin desconocer la importancia de los indicadores para grupos poblacionales específicos, pensamos que desde el enfoque de ciclo vital la mirada es diferente, pues pone la juventud y la vejez en dimensiones del desarrollo que corresponden más bien a consideraciones metodológicas comunes también a nivel socioeconómico y del género.

En el cuadro 1, se presentan las condiciones del desarrollo y los responsables de la protección de acuerdo a los diferentes momentos de vida en la niñez, la adolescencia y juventud. Se trata de un ordenamiento esquemático — con las limitaciones propias de este recurso—, que sigue una secuencia de edades para sistematizar prioridades y modalidades en los esfuerzos de protección integral y fomento del desarrollo. 
CUADRO 1: CICLO DE VIDA Y DESARROLLO EN LA NIÑEZ, ADOLESCENCIA Y JUVENTUD

\begin{tabular}{|c|c|c|c|}
\hline Categoría & Edad & Condición de desarrollo & $\begin{array}{l}\text { Responsables } \\
\text { de la protección }\end{array}$ \\
\hline $\begin{array}{c}\text { Niñez } \\
\text { temprana }\end{array}$ & $\begin{array}{l}0 \text { a } 6 \\
\text { años }\end{array}$ & $\begin{array}{l}\text { Corresponde a la sociedad enfocar los } \\
\text { esfuerzos dirigidos a garantizar un buen } \\
\text { comienzo de la vida que provea de las } \\
\text { necesidades básicas y asegure su supervi- } \\
\text { vencia en un ambiente con condiciones } \\
\text { apropiadas de salud física, mental y seguri- } \\
\text { dad emocional. }\end{array}$ & $\begin{array}{l}\text { Familia. } \\
\text { Estado. }\end{array}$ \\
\hline $\begin{array}{c}\text { Niñez } \\
\text { en edad } \\
\text { escolar }\end{array}$ & $\begin{array}{c}6 \text { a } 12 \\
\text { años }\end{array}$ & $\begin{array}{l}\text { Corresponde enfocar los esfuerzos en } \\
\text { brindar la oportunidad de educación básica } \\
\text { de buena calidad, mantener la protección } \\
\text { de la salud física, mental, seguridad emo- } \\
\text { cional y ampliar el enriquecimiento social. }\end{array}$ & $\begin{array}{l}\text { Familia. } \\
\text { Estado. } \\
\text { Comunidad. } \\
\text { Organizaciones } \\
\text { sociales. } \\
\end{array}$ \\
\hline Adolescencia & $\begin{array}{l}12 \text { a } 18 \\
\text { años }\end{array}$ & $\begin{array}{l}\text { Corresponde el fomento de oportunidades } \\
\text { para desarrollar y orientar capacidades } \\
\text { productivas y reproductivas, impulsar los } \\
\text { medios propicios y seguros para contribuir } \\
\text { y participar en la familia, escuela, comuni- } \\
\text { dad y sociedad, tomando en cuenta sus } \\
\text { opiniones y considerándolo un sujeto acti- } \\
\text { vo y creativo. }\end{array}$ & $\begin{array}{l}\text { Protección y exigibi- } \\
\text { lidad de derechos. } \\
\text { Familia y colabora- } \\
\text { ción intergeneracio- } \\
\text { nal. } \\
\text { Estado y comunidad. } \\
\text { Participación adoles- } \\
\text { cente. }\end{array}$ \\
\hline Juventud & $\begin{array}{c}18 \text { a } 25 \\
\text { años }\end{array}$ & $\begin{array}{l}\text { Corresponde dar continuidad a la experien- } \\
\text { cia acumulada, ampliar la toma de decisio- } \\
\text { nes, encontrar oportunidades para expresar- } \\
\text { se, participar, transferir experiencias a las } \\
\text { nuevas generaciones, contar con capacita- } \\
\text { ción y fortalezas para asumir satisfactoria- } \\
\text { mente su autonomía, la capacidad innova- } \\
\text { dora, proyectos personales y sociales, y } \\
\text { contribuir al desarrollo colectivo. }\end{array}$ & $\begin{array}{l}\text { Ciudadanía plena. } \\
\text { Colaboración inter- } \\
\text { generacional. } \\
\text { Respuesta intersecto- } \\
\text { rial del Estado. }\end{array}$ \\
\hline
\end{tabular}

Fuente: Krauskopf y Muñoz a partir de UNICEF, 2001.

La fase juvenil —adolescencia y juventud- es una de las más desatendidas del ciclo vital, y la invisibilización de los aportes de las personas jóvenes como sujetos sociales contribuye a su visibilización negativa a través de los emergentes comportamentales que conflictúan el orden establecido, entre ellos los Cuatro Jinetes del Apocalipsis: drogas, alcohol, violencia y promiscuidad (Dávila, 2006). Parafraseando a Stigliz et al., cuando dicen que la vejez no es un riesgo en sí misma, podemos decir que la juventud tampoco lo es. Agreguemos que el riesgo para el progreso de las sociedades es su no inclusión en el desarrollo y el ejercicio ciudadano.

La introducción de indicadores específicos de juventud, facilita una correcta visibilización de las realidades juveniles y un mejor sus- 
tento para el desarrollo de políticas públicas pertinentes y equitativas. En este sentido, el aporte de los programas y políticas debe ser evaluado fortaleciendo los enfoques que reconocen al sujeto joven como un actor estratégico, buscan la equidad generacional, de género y etnia para el ejercicio de los derechos, reconocen las importantes relaciones de las juventudes con la democracia y el sistema político, desarrollan la orientación a la inclusión, los canales para la propuesta de iniciativas, el fortalecimiento de las modalidades de institucionalización y la legitimación de la participación con sus formas de conectividad.

\section{PERSPECTIVAS PREDOMINANTES QUE OBSTACULIZAN LA INCORPORACIÓN SOCIAL DE LOS JÓVENES}

Martín-Barbero (1998) destaca que, durante siglos, ser joven se identificó con la negación de la responsabilidad y la productividad, así como lo popular se constituyó por la exclusión de la riqueza, la educación y la cultura. En la misma línea, Lütte concluye que la adolescencia es «una fase de marginación que deriva de las estructuras sociales fundadas en la desigualdad», lo que ocurre en sociedades donde existen claras jerarquías (que incluyen el género), especializaciones del trabajo y diferencias socioeconómicas. Agrega este autor: «nunca se ha probado que los privilegios de la adolescencia requieran de un período de marginación y privación de responsabilidades adultas» (1991:35, 65). Las grandes dificultades que se observan para una real inclusión de los jóvenes en canales de participación y voz pública, en buena medida, tienen como trasfondo la dificultad para aceptar una redistribución generacional del poder político y económico, pues incide en el replanteamiento de las bases de la autoridad para incluir la colaboración intergeneracional en nuestras sociedades.

Las afirmaciones anteriores cuestionan el tradicional concepto de moratoria psicosocial (Erikson, 1974), que desde un enfoque adultocéntrico reconoce a los jóvenes como sujetos carentes de madurez social e inexpertos, incluyéndolos predominantemente como un pre-proyecto del adulto de mañana (Krauskopf, 2004). Las políticas que se basan en estrategias derivadas del paradigma del futuro esperable - encarnado en la adultez actual como foco orientador de la preparación de la juventud - ven reducida su efectividad ante la falta de predictibilidad que se desprende de la interacción rápida de factores a escala mundial con la moderna prolongación de la vida. Esto se acentúa aún más en el desarrollo de los jóvenes que crecen en exclusión, donde la prolonga- 
ción de la vida no constituye una promesa existencial, las gratificaciones efímeras (muchas veces riesgosas) toman predominio y se aprecia la premura psicosocial en lugar de la moratoria.

Salazar ha identificado la cultura de muerte, donde se acortan las distancias entre vivir y morir para los jóvenes sicarios, los miembros de las maras ${ }^{1}$, etcétera. Cita a Perea: «Son los jóvenes destruyendo su propio entorno, su propia generación y los referentes de su identidad perdida» (1998:164). Reguillo (1999) concuerda al señalar que, para los que no hay opciones y son mirados como desechables, la muerte se convierte en una experiencia más fuerte que la vida.

El concepto de moratoria psicosocial está presente en nuestro imaginario social, contribuye a postergar la responsabilidad ciudadana y favorece la discriminación por edad que afecta a los jóvenes. La resistencia a través de la desconexión activa es una expresión importante ante la ausencia de propuestas realmente incluyentes para las juventudes. Surgen la desafección partidaria, las autoorganizaciones — bandas y pandillas — que giran en torno a la transgresión social y la violencia como empoderamiento aterrante frente a la invisibilización y exclusión (Krauskopf, 1996). Son condiciones nocivas tanto para los mismos jóvenes como para la calidad de la vida social en su conjunto, partiendo por la democracia.

\section{EL PROBLEMA DE LAS EDADES PARA EL USO DE INDICADORES DE JUVENTUD}

Las sociedades modernas abordan el desarrollo de las personas por rangos etáreos en las políticas correspondientes. La edad, como señalamos, es un dato necesario pero no suficiente y tiene valores distintos en sociedades, estratos socioeconómicos, culturas. Por otro lado, si bien dominan los criterios etáreos en los indicadores referidos a la juventud en la política pública, estos criterios son contradictorios, puesto que no existe un enfoque de ciclo vital que sea congruente con los procesos de avance que se desarrollan en cada etapa. Es el caso de la legislación y las instituciones de protección, salud y sexualidad. La nueva legislación penal juvenil tiende a fijar una edad límite por debajo de la cual no se considera responsable de sus actos a la persona, pero los países tienen límites de edad diferentes. A partir de los 18

1 Término que surge en Centroamérica, originariamente en Guatemala, para designar las pandillas juveniles. 
años, las personas son juzgadas como adultas, reproduciendo el problema de la ambigüedad en el reconocimiento del sujeto juvenil, en virtud del carácter dual de su condición sociojurídica, que favorece su invisibilización en la planificación social (Krauskopf, 2003b).

En 1985, la Asamblea General de las Naciones Unidas para el Año Internacional de la Juventud, adoptó el límite de catorce años para definir la niñez y el límite de quince a veinticuatro años inclusive, para definir la juventud. En esta última categoría se hizo la distinción de los adultos jóvenes, entre veinte y veinticuatro años. La OMS define adolescencia desde los diez a los diecinueve años once meses - lo que coincide con las edades aproximadas en que se inician las modificaciones sexuales y que marcan la culminación de este crecimiento- $-\mathrm{y}$ la juventud, desde los quince a los veinticuatro años con once meses. La Convención de los Derechos del Niño establece la niñez hasta los dieciocho años y los Códigos derivados de ella ubican la niñez hasta los doce años y la adolescencia hasta los dieciocho años. Para la Convención Iberoamericana de los Derechos de los Jóvenes, la juventud abarca desde los dieciocho a los veinticuatro años (Krauskopf, 2003a).

Vemos que el discurso sobre el sujeto joven presenta la superposición etaria de la adolescencia y la juventud. Se considera que la juventud engloba la adolescencia, pero la adolescencia no engloba a la juventud. Esto tiene consecuencias en la forma en que se concretan las políticas: se da una existencia predominante a programas de adolescencia que contribuye al desarrollo de la juventud, pero no se alcanzan períodos cruciales de la vida de las personas jóvenes, las que quedan subsumidas en la categoría de adulto (Krauskopf, 2003a). Nuestra propuesta es incluir adolescencia y juventud como fase juvenil, la cual indudablemente tiene sub etapas.

En la mayor parte de las legislaciones, las personas se consideran adolescentes antes de los dieciocho años y luego son adultas. En consecuencia, a partir de los dieciocho años adquieren el estatus de la ciudadanía formal. Sin embargo, también se dan países como Nicaragua y Ecuador donde se adquiere la capacidad de votar a los dieciséis años. La reducción de la edad para votar a dieciséis años está siendo estudiada en varios países como en el caso de la Argentina, Colombia, Brasil, Perú, Venezuela. La entrega de la cédula de identidad plena también tiene variaciones en edad.

El panorama se hace confuso. Las particularidades de las juventudes tienden a diluirse en un diseño sectorial de política social, eco- 
nómica y laboral que se dirige al conjunto de la población adulta sin distingo alguno. Aparecen algunos programas específicos, aislados y compensatorios - frecuentemente trabajo juvenil y salud adolescente-, donde también se aprecia el problema de las edades. Las políticas de juventud de diversos países apuntan a límites de edad inferiores y superiores diversos. Los registros por edad de los sectores de la administración gubernamental no se encuentran organizados en rangos similares. Todo ello fragmenta los criterios para la atención del desarrollo, se pierden inversiones que se han hecho en una etapa al no considerar políticas de continuidad y profundización para la siguiente, y se evidencia un vacío imprescindible de superar para el desarrollo de indicadores efectivos de juventud.

\section{ENFOQUES HABITUALES QUE RESTRINGEN EL PROGRESO DE LAS JUVENTUDES}

La frecuente visión de la juventud - y adolescencia - como período preparatorio de seres en transición involucra la postergación de la acción y la subordinación del aporte participativo del sujeto juvenil ciudadano. Falta el reconocimiento de que los jóvenes están asentándose en el presente y encarnan los cambios más fuertes de la modernidad. Requieren validar su calidad de sujetos sociales y proyectan su futuro a partir del sentido presente de sus vidas (Krauskopf, 2003b).

Las políticas tradicionales marginan las realidades juveniles del desarrollo nacional, por lo que aíslan las acciones específicas para la juventud del resto del aparato del Estado. Esto se refleja en las funciones de los Organismos Oficiales de Juventud, su escasa vinculación a los Sectores del Estado y a los niveles superiores de la jerarquía gubernamental. Agreguemos que en América Latina la institucionalidad encargada de la juventud es generalmente la más débil en la estructura administrativa de los gobiernos.

El enfoque tradicional de las políticas da lugar a representaciones sociales desvinculadas de la experiencia y al sentido de la cosmovisión juvenil. Los jóvenes son vistos como generación de relevo de los adultos y se considera que sólo al alcanzar la adultez llegarían a ser sujetos sociales. La meta es el adulto traducido por los estereotipos sociales. Se trata de un enfoque adultocéntrico y una relación adultista. Sin embargo, paradojalmente, tampoco el adulto actual corresponde a identidades fijas. Las distancias generacionales con los adultos se han modificado. Ahora los jóvenes saben cosas que los adultos no 
saben. Esto hace que la participación juvenil no sólo sea un claro derecho, sino que también es una parte insustituible del conocimiento de las situaciones y alternativas (Krauskopf, 2003a).

Entre las políticas que inciden en la situación de las juventudes, son muy importantes aquéllas implícitas, en las que el joven no es sujeto social; se sitúan en el entrenamiento para la defensa de la nación como es el servicio militar o en la preparación a la inserción social adulta, como es el caso de la educación. El ámbito educacional restringe su perspectiva a alumnos o educandos y tiene dificultades para integrar la convivencia de sujetos sociales con inquietudes y expectativas propias. Diversos autores han enfatizado que una reconsideración del progreso implica una reconsideración del valor de la educación, así como el acceso del individuo a una educación de excelencia.

La prevalencia de políticas implícitas, omisas y aisladas mantiene la invisibilidad del sujeto juvenil, el que pasa a visibilizarse cuando altera el orden social. Así la juventud, que desde el paradigma de preparación es vista como la promesa del futuro, pasa a ser vista como la amenaza del presente. Surgen medidas coyunturales provocadas por un sentido de urgencia en diversas situaciones emergentes, que frecuentemente apelan al control y la represión social para brindar una imagen de protección a la sociedad, con lo que contradicen planteamientos oficiales que se declaran respetuosos de los derechos.

La carencia de ámbitos para una visibilidad positiva y para la incorporación social de las habilidades y destrezas de niños y jóvenes excluidos pueden hacer de la calle, la escuela y la cárcel, la universidad (Sosa y Rocha, 2001). La visibilidad aterrante se torna en opción de empoderamiento y las gratificaciones intensas son más importantes que la preservación de la vida (Krauskopf, 1996). A partir de la visibilización negativa emergen respuestas de política pública para tratar el déficit, el riesgo y la transgresión juvenil con perspectivas reactivas y reduccionistas. La juventud es vista como etapa problema.

En este enfoque, la atención se presta a problemas específicos, se desarrolla desde políticas sectoriales y compensatorias y los grupos meta relevantes se encuentran en la juventud urbana popular. Se traduce en programas orientados a los comportamientos de riesgo y transgresión, su asistencia y prevención, así como programaciones que se inspiran en matrices de corte autoritario para la rehabilitación de jóvenes. Las acciones policíacas e incluso del ejército, orientadas al control de la delincuencia recaen fuertemente en los jóvenes. En países donde prevalece el populismo punitivo para enfrentar a los jóvenes 
(Santa Cruz, 2003), parece darse un debilitamiento de las posibilidades de desarrollar estrategias sostenibles para la implementación de políticas avanzadas de juventud.

\section{ENFOQUES INCLUYENTES QUE FAVORECEN LA INCORPORACIÓN Y CALIDAD DE VIDA DE LAS JUVENTUDES}

En las sociedades contemporáneas la fase juvenil ocupa en el ciclo vital un número creciente de años, la pubertad aparece a edades más tempranas y el ingreso a la adultez ya no se da en forma lineal ni por secuencias ordenadas. Las características biopsicosociales del período juvenil facilitan la liberación de energías psíquicas, lo que a su vez acelera el cambio. La vida adulta se prolonga en el contexto de la velocidad de los cambios por lo que no es un período estable, sino que está sujeta a importantes transformaciones. Los cambios puberales son un punto de partida para asumir un nuevo rol social y construir la propia identidad: emerge el ser productor y reproductor y los jóvenes cuentan, por primera vez, con la propia capacidad para aportar a la conducción de su proceso existencial, completar vacíos que se dieron en las bases de su experiencia de vida y reenfocar situaciones, tanto de la niñez como de su presente.

Las sociedades de la era moderna requieren contar con individuos capaces de aprender a aprender y reciclar con flexibilidad sus competencias y actitudes. La elaboración de identidades encuentra nuevos canales en el espacio cibernético, aparecen expresiones culturales y conocimientos manejados ágilmente por las nuevas generaciones. Los jóvenes de los últimos decenios han crecido en un ecosistema comunicativo tecnológico, enfrentan con facilidad la obsolescencia del conocimiento y aportan respuestas innovadoras. La experiencia ya no es el único referente para ampliar el conocimiento y los adultos también se ven impelidos a flexibilizar sus procesos identitarios para incorporar las nuevas transiciones del mundo contemporáneo, en el contexto de la prolongación de sus vidas. Hoy, con mayor fuerza que en el pasado, las juventudes son un eje central en las nuevas estrategias de desarrollo (Rama, 1992, citado en Bango, 1997).

El enfoque de las responsabilidades estatales ha experimentado cambios al enfatizarse nuevos parámetros, donde cabe destacar la inclusión prioritaria de los derechos humanos con el fortalecimiento - entre otros- de las identidades sociales de niños, mujeres, etnias y juventudes. Como consecuencia de ello, a las políticas de sectores ha debido agregarse la construcción de políticas de actores. Este cambio históri- 
co tiene respaldo en la actual inclusión democrática de niños, adolescentes y jóvenes en la Convención de los Derechos de la Niñez (1998) y la reciente Convención Iberoamericana de los Derechos de los Jóvenes (OIJ, 2005) ratificada por siete países de Iberoamérica.

Desde la Doctrina de la Protección Integral se actúa brindando protección de los derechos, en lugar de proteger a los niños y adolescentes en tanto personas como lo plantea la Doctrina del Menor Irregular. Se trata de una protección y prevención apoyada en el empoderamiento que deriva de la promoción de condiciones para la capacidad de decisión y de participación. La protección social debe reconocer que el retiro de respuestas inadecuadas requiere ir acompañado de la oferta de un replanteamiento de la percepción de los problemas y opciones, junto con respuestas más satisfactorias a las necesidades de las poblaciones vulnerables. Además, se considera la restitución de los derechos que han sido violados, lo que desemboca, por lo tanto, en acciones reparatorias.

La acumulación de experiencias y la sistematización de conocimientos evidencian que la implementación de estos avances requiere precisiones en el concepto de participación para ir de la apariencia a la efectividad y al desarrollo de estrategias intergeneracionales de colaboración. Ya no se trata sólo de la ciudadanía formal de ejercer el derecho al voto, sino que se da centralidad al desarrollo de la ciudadanía integral de niños, adolescentes y jóvenes. El reconocimiento del aporte productivo y cultural de los jóvenes y su papel como actores estratégicos del desarrollo, se traduce en programas que incluyen procesos de integración social para los jóvenes y fomentan el ejercicio de la ciudadanía. La participación social y el ejercicio ciudadano reintegran a las juventudes al campo político, restablecen el vínculo social y aportan a la cohesión y gobernabilidad democrática. Aún cuando se observa que diversas organizaciones y gobiernos reconocen que las juventudes requieren participar en los procesos sociales que afectan sus vidas, en los hechos, las acciones que incluyen dicha participación son todavía escasas (Krauskopf, 2008).

Un producto de esta nueva mirada sobre el papel de las juventudes en el progreso de sus sociedades se refleja en la importancia que últimamente se ha asignado como bono demográfico (Banco Mundial, 2007). En muchos países de América Latina, la pirámide demográfica todavía no se ha revertido, constituyéndose la población adolescente y juvenil en la ventana de oportunidad para un bono demográfico. Así, el incremento de las inversiones en el rescate del capital humano juvenil es una estrategia para resolver problemas del desarrollo en países 
que luego afrontarán una capa creciente de población mayor de edad, la que demandará más servicios y dependerá del aporte de los jóvenes de hoy a sus sociedades.De su participación socioeconómica dependerá cada vez más la creciente población envejecida, como también la posibilidad de aportar para su propio desarrollo.

\section{LA CONSTRUCCIÓN DE INDICADORES DE CALIDAD DE VIDA DE LOS JÓVENES}

Apuntamos a indicadores que midan el grado, distribución y avance del bienestar de los jóvenes como parte de la calidad de vida, para lo cual son fundamentales las condiciones de inclusión que permitan el desarrollo humano y la ciudadanía y que son producto de la interacción entre las circunstancias sociales, políticas, económicas y culturales que afectan a los jóvenes y las colectividades a que pertenecen. Concordamos con Buvinic (1981) en que estos indicadores apuntan a problemas sociales y son políticos: los valores y metas colectivas orientan su definición. Por ello, en nuestra propuesta pensamos que corresponde elaborar tanto indicadores cuantitativos como cualitativos. Así se tendrá información sobre cuán cerca o lejos están las condiciones de vida de los jóvenes de las metas o normas establecidas, los alcances de la intervención sociopolítica, además de valorar el bienestar subjetivo de las personas jóvenes medido con base en sus propios juicios, opiniones y experiencias. Los dos tipos de indicadores son complementarios. Un ejemplo lo encontramos en las medidas objetivas como la incidencia juvenil al no participar en elecciones y las subjetivas, como las razones de los jóvenes para no votar.

Un aspecto metodológico es el enfoque generacional introducido en algunos instrumentos sobre salud en la calidad de vida de los adolescentes, que se traduce en la participación de éstos en la construcción de los indicadores (Hidalgo, 2008). Cuando los indicadores son dirigidos a la población general, son construidos por adultos y generalmente respondidos por éstos. En el caso de la fase juvenil, se necesitan los saberes de las personas jóvenes sobre las implicancias que tiene para ellos la calidad de vida así como el lenguaje en que decodifican los estímulos. Se reconoce así una diferencia sustantiva que parte del concepto de ciclo de vida y procura el necesario aporte de la colaboración intergeneracional en la construcción de conocimientos sobre juventud.

La situación de los jóvenes ha sido medida a través de censos, encuestas o inventarios que clasifican principalmente sus características y 
pertenencias (género, etnia, rural, urbano, etcétera). Los indicadores sobre las juventudes en el progreso de las sociedades requieren ser el resultado de una selección y elaboración específica e intencionada de las estadísticas disponibles. Consideramos que deben aplicarse aquéllos que revelen la realidad detrás de los números, develen diferencias o disparidades, muestren los alcances de las acciones públicas y sigan el progreso en la erradicación de desigualdades, así como los avances de la inclusión de los jóvenes en sus sociedades. Dada la complejidad de estas condiciones queremos contribuir con la propuesta de algunos lineamientos que permitan la elaboración de indicadores como señales para mirar la calidad de los jóvenes en sus sociedades.

De acuerdo a lo que hemos expuesto a lo largo de este trabajo, proponemos incluir indicadores que informen sobre los siguientes ámbitos.

i) Indicadores de situación, ${ }^{2}$ por ciclo de vida y grupos específicos, principalmente aquellos afectados por: desigualdad, ${ }^{3}$ polarización socieconómica, género, culturas y necesidades especiales. Considerar: ventana de oportunidad para el bono demográfico y posible análisis por quinquenios de edad.

ii) Indicadores de políticas ${ }^{4}$

Indicadores de actores: política de juventud, participación ciudadana y prácticas discriminatorias.

Indicadores de sectores: Enfoque ${ }^{5}$ de inclusión juvenil de los sectores de administración del Estado en educación, salud, trabajo, ${ }^{6}$ cultura, protección social, justicia.

2 Fundamental es el análisis social que se haga de los resultados de los indicadores, esto es, para explorar la relación entre la situación de vida de las juventudes y las causas histórico-políticas, sociales y económicas que la determinan.

3 Contribuir a promover la igualdad de oportunidades.

4 Se valora la intervención social, al definir objetivos de políticas y metas de programas que promueven el desarrollo y el bienestar, así como evaluar los efectos de la acción social intencionada.

5 Proponemos los enfoques: período preparatorio, etapa problema, sujeto de derechos, actor estratégico del desarrollo, ciclo de vida.

6 Se ha constatado que el desempleo tiene grandes efectos deteriorativos en el bienestar de los individuos y de las naciones. Los jóvenes frecuentemente lideran este tipo de estadísticas, particularmente en períodos de crisis. 
iii) Legislación: ley de juventud; ley penal juvenil; otras leyes que inciden en la vida juvenil: laborales, electorales, servicio militar, etc.; implementación de la legislación.

iv) Institucionalidad: ${ }^{7}$ jerarquía de la institucionalidad de juventud, ${ }^{8}$ dependencia administrativa de la institucionalidad de juventud, presupuesto y estabilidad de los programas en juventud, coordinación intersectorial en juventud.

La Política de Juventud puede ser el principal instrumento de referencia de un sistema de acciones dirigidas a la juventud, donde existen múltiples componentes que dan cuenta de una común orientación. Para su análisis consideramos la importancia de contar con indicadores de coherencia y aplicación, entre los que destacamos: ${ }^{9}$

i) Una representación sobre la juventud y su realidad ciudadana, exenta de estereotipos y discriminación.

ii) Un marco normativo que propicia y respalda la realización de la condición de ciudadanía en las y los jóvenes. Frecuentemente una Ley de Juventud que puede contribuir a afirmar una Política de Estado incluyente de la juventud.

iii) Una Ley de Juventud que especifica el desarrollo de las políticas. Es importante el carácter vinculante de la Ley, el alto nivel que asigne a la Instancia de juventud para la coordinación de la Política y un presupuesto acorde con el desarrollo de políticas.

iv) Un marco orientador para las ofertas programáticas que garantizan el ejercicio de los derechos sociales, culturales y económicos de la juventud, mediante modelos de gestión abiertos a la participación de los propios jóvenes y de su entorno social de forma género sensitiva.

7 Se valora el buen funcionamiento de las instituciones y sus soluciones: las políticas y programas de intervención.

8 Un elemento adicional que merece ser analizado es cuando el criterio etáreo y de adscripción política de los miembros clave de estos organismos de juventud, desplaza la capacidad técnica. Ello puede ocurrir en asociación con un débil respaldo gubernamental al desarrollo de las políticas de juventud. En estos casos no se garantiza el capital técnico y cultural requerido para las complejas y pioneras funciones que deben cumplir los organismos de juventud.

9 Ampliado y basado en la Política Pública de la Persona Joven de Costa Rica, 2003. 
v) La construcción y coordinación de políticas a nivel local y sectorial con claridad respecto a las asignaciones presupuestarias.

vi) Una política de sectores específicos que debe ser explicitada y formalizada de acuerdo a las realidades propias del sector en el marco de las directrices de la política nacional y local para articular coherentemente los diferentes proyectos intersectoriales y con la sociedad civil.

vii) Mecanismos institucionales y de gestión con las y los jóvenes, sustentados en una rectoría clara, con alto rango, capaz de fijar horizontes y desconcentrar permanentemente las acciones y las responsabilidades.

viii) Un conjunto articulado de mecanismos de comunicación pública que permita: a) la información sobre la situación, normativa y oportunidades existentes en materia de desarrollo juvenil; b) conocer el funcionamiento de la ley y la política pública de juventud; c) la visibilidad de las iniciativas; d) la escrutabilidad ciudadana respecto del uso de los recursos, los resultados y la evaluación de las acciones.

Se hace necesario construir indicadores para medir la presencia y características de la participación juvenil y darle seguimiento. Deben evaluar el papel de los jóvenes como actores de cogestión para enriquecer el espacio para la acción pública y el desarrollo democrático. Se pueden señalar tres componentes en el proceso participativo: el poder político, regulador de los cauces de participación y de la ejecución de los acuerdos; la planificación, o personal técnico encargado de proponer y recibir alternativas de parte de los jóvenes, y la población, con derecho a la información y formación para ejercer la participación desde el reconocimiento de las auténticas formas constructivas que los jóvenes viven y proponen.

En los proyectos y programas existentes se ha podido apreciar que la inclusión participativa presenta una gradiente de compromiso y empoderamiento (Krauskopf, 2008) en que los jóvenes:

i) Tienen una presencia decorativa.

ii) Tiene una presencia simbólica y testimonial.

iii) Reciben y proveen información con retroalimentación para mejorar objetivos y resultados.

iv) Son consultados para establecer, priorizar y definir objetivos, toman decisiones, se coordinan con los demás participantes y son coresponsables de los resultados.

v) Inician la acción, desarrollan movilizaciones, proyectos y propuestas propias, fijan objetivos, metodologías, se expresan si es nece- 
sario con sus códigos, buscan apoyo, asesoría, acompañamiento adulto cuando lo requieren.

El estudio de los indicadores de la calidad de vida en la juventud es relativamente reciente y ha partido con mayor fuerza derivado de la preocupación por la adolescencia desde el campo de la salud. Esto ha sido posible desde la reunión de Alma Ata donde se abandonó la definición de salud como ausencia de enfermedades, lo que dio lugar a transformaciones de los paradigmas que tradicionalmente definían la salud de modo residual (Krauskopf, 2003a). La participación social emerge como estrategia en el campo de la salud con la modernización del aparato estatal y el fortalecimiento del sistema democrático, con el fin de alcanzar el bienestar, la inclusión e integración social. Estos enfoques de la OMS/OPS contribuyen a la introducción pionera de programas y políticas dirigidos a la salud integral de la adolescencia y juventud en América Latina y el Caribe. Llamativamente, esto no ocurre en el campo de la educación, considerada la política por excelencia para el desarrollo de las jóvenes generaciones.

\section{CONSIDERACIONES FINALES}

En el siglo XXI, la articulación de las fuentes de la identidad se sustenta cada vez menos en la linealidad del desarrollo y las discriminaciones asentadas en el patriarcado. Ello conlleva la necesidad de hacer frente a la redistribución del poder, que implica la participación juvenil auténtica, la equidad de género, los canales para una colaboración intergeneracional que valoriza a la juventud ciudadana, su flexibilidad para incorporar los conocimientos y aportar a la innovación.

Los obstáculos para el progreso de la calidad de vida de las juventudes se encuentran vinculados a dificultades presentes en las sociedades latinoamericanas para el reconocimiento de la contribución de las juventudes al desarrollo socioeconómico, así como la débil presencia de políticas y programas de juventud que aporten a su integración social. Es necesario desarrollar revisiones y metodologías que vayan mas allá de las descriptivas, que son las más frecuentes en relación a juventud, y que incorporen metodologías explicativas y prospectivas con indicadores no sólo de valor técnico, sino también político, para permitir la definición de prioridades en la meta de la inclusión social de las juventudes en el progreso. 
El posicionamiento de las juventudes en el progreso de las sociedades se refleja en indicadores de calidad de vida que valoren el avance de las relaciones de colaboración entre las generaciones y apunten al establecimiento de vínculos que fortalecen el lazo social y la cohesión, a la reducción de las brechas de desigualdad con una perspectiva de equidad en el enfrentamiento de las dificultades que generan el fraccionamiento de las sociedades, la exclusión y la pobreza. Es necesaria una redemocratización de espacios con la inclusión de los jóvenes y sus comunidades, fomentando la responsabilidad de los sujetos con sus entornos más directos e inmediatos, la identificación de problemas, necesidades y posibles soluciones propias. No hacerlo, profundiza el conflicto social, obstaculiza el desarrollo juvenil y su aporte estratégico al progreso de sus sociedades.

La calidad de vida es producto de múltiples dimensiones y se constituye en la meta social ineludible. Para lograr el progreso en las sociedades y medirlo es necesario considerar la inclusión de las necesidades, capacidades y oportunidades de los y las jóvenes en todas las políticas públicas. Esto involucra analizar los resultados de la política y de la acción social con un claro enfrentamiento de los obstáculos al desarrollo juvenil, expresado en la articulación de las políticas públicas con un enfoque de transversalidad, equidad y sostenibilidad para favorecer las dimensiones clave de la inclusión de las personas jóvenes en la sociedad.

SANTIAGO (CHILE), ENERO 2011

RECIBIDO: FEBRERO 2011

ACEPTADO: MARZO 2011

\section{REFERENCIAS BIBLIOGRÁFICAS}

BANCO MunDiAL (2007): «Informe sobre el desarrollo mundial. El desarrollo y la próxima generación». Washington: BM.

BANGO, Julio (1997): «Las políticas de juventud al final del milenio». Madrid: Organización Iberoamericana de la Juventud.

DÁVILA, ÓsCAR (2006): «Estudiantes secundarios y su lucha por la igualdad educativa y social». Viña del Mar: CIDPA.

Erikson, Eric (1974): Identidad, juventud y crisis. Buenos Aires: Paidós.

MAYRA, BUVINIC (1981) Women and development: Indicators of their changing role. Unesco. Disponible en: www.siise.gov.ec. 
CEPAL/OIJ (2008): «Juventud y cohesión social en Iberoamérica». Santiago: CEPAL/OIJ.

CPJ (2003): «Política pública de la persona joven». San José: Consejo Nacional de Política Pública de la Persona Joven.

HART, Roger (1997): Children's Participation in Sustainable Development: The Theory and Practice of Involving Young Citizens in Community, Development and Environmental Care. London: Earthscan Publications Limited.

Hidalgo, Alejandro (2008): De los comportamientos de riesgo a la calidad de vida de los adolescentes. México: Ed. Universidad de Guadalajara.

KRAUSKOPF, DinA (2008a): «Dimensiones de la participación en las juventudes contemporáneas latinoamericanas». Revista Pensamiento Iberoamericano (3). Madrid.

- (2008b): «Avances y desafíos en la institucionalidad pública en juventud». Revista Observatorio de Juventud. Santiago: INJUV.

- (2004): «Comprensión de la juventud. El ocaso del concepto de moratoria psicosocial». JOVENes, Revista de Estudios sobre Juventud. Nueva Época, N²1. México: IMJ.

_ (2003a): «Participación social y desarrollo en la adolescencia». San José: UNFPA.

(2003b): «La construcción de políticas de juventud en Centroamérica». ÓsCAR DÁviLA (editor): Políticas públicas de juventud en América Latina: políticas nacionales. Viña del Mar: Ediciones CIDPA.

- (1996): «Violencia juvenil: alerta social». Revista Parlamentaria. La crisis social: desintegración familiar, valores y violencia social. San José, 4 (3).

LÜTTE, G. (1991): Liberar la adolescencia. La psicología de los jóvenes de hoy. Barcelona: Herder.

MARTÍN-BARBERO, JeSÚS (1998): «Jóvenes: des-orden cultural y palimpsestos». Humberto CUBIDES, MARÍA CRISTINA LAVERDE y CARLOS EDUARDO VALDERRAMA (editores): Viviendo a toda: jóvenes, territorios culturales y nuevas sensibilidades. Santa Fe de Bogotá: Siglo de Hombre Editores, Departamento de Investigaciones Universidad Central.

OIJ (2005): «Convención iberoamericana de los derechos de la juventud». Madrid: OIJ.

Reguillo, Rossana (1999): «La ética de lo efímero o los compromisos itinerantes». Apuntes. Lima: Casa de la Juventud.

SAlAZAR, AlONSO (1998): «Juventud y violencia». JAIME ARTURO PADILLA (compilador): La constitución de lo juvenil. México: Editorial Causa Joven.

SANTA CruZ, MARÍA (2003): «Violencia y juventud en El Salvador». Ponencia presentada en la Conferencia Centroamericana de Juventud. Antigua: CIRMA, Rockefeller Foundation y Fundación Frederich Ebert. 
SOSA, JUAN JosÉ y JosÉ LUIS Rocha (2001): «Las pandillas en Nicaragua». En: Maras y pandillas en Centroamérica. Volumen 1. Managua: Publicaciones UCA.

StiglitZ, Joseph, AMARTYA Sen y JeAn-PAul Fitoussi (S/F): Report by the Commission on the Measurement of Economic Performance and Social Progress. Disponible en: www.stiglitz-sen-fitoussi.fr.

UNICEF (2001): «Adolescencia en América Latina y el Caribe: orientaciones para la formulación de políticas». Nueva York: UNICEF. 